\title{
RUSSO'S FORMULA, UNIQUENESS OF THE INFINITE CLUSTER, AND CONTINUOUS DIFFERENTIABILITY OF FREE ENERGY FOR CONTINUUM PERCOLATION
}

\author{
JIANPING JIANG, ${ }^{*}$ \\ SANGUO ZHANG ${ }^{* * * * * *}$ AND \\ TIANDE GUO, ${ }^{* *} * * * *$ Graduate University of Chinese Academy of Sciences
}

\begin{abstract}
A new formula for continuum percolation on the Euclidean space $\mathbb{R}^{d}(d \geq 2)$, which is analogous to Russo's formula for bond or site percolation, is proved. Using this formula, we prove the equivalence between uniqueness of the infinite cluster and continuous differentiability of the mean number of clusters per Poisson point (or free energy). This yields a new proof for uniqueness of the infinite cluster since the continuous differentiability of free energy has been proved by Bezuidenhout, Grimmett and Löffler (1998); a consequence of this new proof gives the continuity of connectivity functions.
\end{abstract}

Keywords: Continuum percolation; Russo's formula; uniqueness of the infinite cluster; free energy; connectivity function

2010 Mathematics Subject Classification: Primary 60K35

Secondary 82B43; 60G55

\section{Introduction}

Russo's formula first appeared in [7] and was rediscovered by Russo in [13] for site percolation. This formula proved to be very useful in studying properties of bond or site percolation (see, for example, [5, Section 2.4] and [6, Section 4.7]). In [15], Zuev derived an analogous formula for Poisson random fields. It is easy to apply the formula in [15] to the probability of an event in continuum percolation. With the idea from [15], we derive Russo's formula for the expectation of a random variable in continuum percolation. The extension of the formula from the probability of an event to the expectation of a random variable in continuum percolation is not straightforward since the number of sites in a bounded domain is not bounded in continuum percolation.

Aizenman et al. [1] presented results dealing with three related issues in bond or site percolation theory: (a) uniqueness of the infinite cluster, (b) continuity of the connectivity functions, and (c) continuous differentiability of the mean number of clusters per site. They proved the equivalence between (a) and (c), established (c), and then proved (b) by (a). In continuum percolation, (a) was proved by Meester and Roy [8] and (c) was proved by

\footnotetext{
Received 23 November 2009; revision received 5 April 2011.

* Current address: Department of Mathematics, University of Arizona, Tucson, Arizona 85721, USA.

Email address: jiangjianping11@gmail.com

** Postal address: School of Mathematical Sciences, Graduate University of Chinese Academy of Sciences, 100049,

Beijing, P. R. China.

*** Email address: sgzhang@gucas.ac.cn

**** Email address: tdguo@gucas.ac.cn
} 
Bezuidenhout et al. [2]. In this paper we prove results analogous to those in [1] (except for (c)) for continuum percolation.

Next, we introduce the setup for our main results. The set of real numbers is denoted by $\mathbb{R}$, and the set of natural numbers $\{1,2, \ldots\}$ is denoted by $\mathbb{N}$. Given a point set $\mathcal{X} \subset \mathbb{R}^{d}$, we denote by $G(\mathcal{X}, 1)$ the undirected graph with vertex set $\mathcal{X}$ and undirected edges connecting all point pairs $\{x, y\}$ with $\|x-y\| \leq 1$. In this paper we restrict attention to the case where $\|\cdot\|$ is the Euclidean norm ( $l_{2}$ norm), but usually its generalizations to other norms are straightforward. Also, we use the same norm to define the diameter of subsets of $\mathbb{R}^{d}$, that is, for $A \subseteq \mathbb{R}^{d}$, we set

$$
\operatorname{diam}(A):=\sup \{\|x-y\|: x \in A, y \in A\},
$$

where ':=' denotes a definition but '=' can also denote a definition when the context is clear. The Lebesgue measure in $\mathbb{R}^{d}$ is denoted by $\ell(\cdot)$. For any finite or countable subset $A$ of $\mathbb{R}^{d}$, we write $|A|$ for the cardinality (number of elements) of $A$.

Let $\Omega$ denote the set of all countable subsets of $\mathbb{R}^{d}$. We call an element of $w \in \Omega$ a configuration, and, abusing the terminology slightly, we call points of $w \in \Omega$ Poisson points. For any nonnegative measurable function $g$ on $\mathbb{R}^{d}$, we let $\mathrm{P}_{g}$ denote the probability measure on $\Omega$ associated with the nonhomogeneous Poisson point process with intensity $g$; we write $\mathrm{E}_{g}$ for the corresponding expectation. In the special case of a homogeneous Poisson process $g \equiv \lambda$, we write $\mathrm{P}_{\lambda}$ and $\mathrm{E}_{\lambda}$. We write $\mathscr{H}_{\lambda}$ for a homogeneous Poisson process of intensity $\lambda$ on $\mathbb{R}^{d}$, i.e. $\mathrm{P}_{\lambda}$-distributed random elements of $\Omega$. A component (or cluster) of a graph $G$ is a maximal connected subgraph of $G$. Continuum percolation can loosely be characterized as the study of large clusters of the infinite random graph $G\left(\mathscr{H}_{\lambda}, 1\right)$. Equivalently, one may study the connected components of the union of balls of radius $\frac{1}{2}$ with centers in $\mathscr{H}_{\lambda}$ (see [10, Chapter 10] for more details).

Let $\mathscr{H}_{\lambda, 0}$ denote the point process $\{\boldsymbol{0}\} \cup H_{\lambda}$, where $\mathbf{0}$ is the origin in $\mathbb{R}^{d}$. For $k \in \mathbb{N}$, let $p_{k}(\lambda)$ denote the probability that the component of $G\left(\mathscr{H}_{\lambda, 0} ; 1\right)$ containing the origin is of order $k$ (here order means the number of Poisson points in this component). The percolation probability $p_{\infty}(\lambda)$ is the probability that 0 lies in an infinite component of the graph $G\left(\mathscr{H}_{\lambda, 0} ; 1\right)$, and is defined by

$$
p_{\infty}(\lambda)=1-\sum_{k=1}^{\infty} p_{k}(\lambda) .
$$

The critical intensity $\lambda_{\mathrm{c}}$ is defined by

$$
\lambda_{\mathrm{c}}=\inf \left\{\lambda>0: p_{\infty}(\lambda)>0\right\} .
$$

The value of $\lambda_{\mathrm{c}}$ depends on the dimension $d$. The fundamental result of continuum percolation says that $0<\lambda_{\mathrm{c}}<\infty$, provided that $d \geq 2$; see [5, Theorem 12.35] or [9, Theorem 3.3].

For $x, y \in \mathbb{R}^{d}$, we say that $x$ and $y$ are connected through $\mathscr{H}_{\lambda}$ and write $x \leftrightarrow y$ through $\mathscr{H}_{\lambda}$ if there exists a sequence of Poisson points $u_{1}, u_{2}, \ldots, u_{m}$ of $\mathscr{H}_{\lambda}$ such that $\left\|x-u_{1}\right\| \leq 1$, $\left\|u_{i}-u_{i+1}\right\| \leq 1, i=1,2, \ldots, m-1$, and $\left\|u_{m}-y\right\| \leq 1$. For $A, B \subset \mathbb{R}^{d}$, we say that $A$ and $B$ are connected through $\mathscr{H}_{\lambda}$ and write $A \leftrightarrow B$ through $\mathscr{H}_{\lambda}$ if there exist $x \in A$ and $y \in B$ such that $x \leftrightarrow y$ through $\mathscr{H}_{\lambda}$ (we write $A \leftrightarrow B$ if no such $x$ and $y$ exist). The (two-point) connectivity function $\tau(x, y)$ is the probability that $x$ and $y$ are connected through $\mathscr{H}_{\lambda}$. Similarly, for $x_{1}, \ldots, x_{n} \in \mathbb{R}^{d}$, the $n$-point connectivity function, $\tau\left(x_{1}, \ldots, x_{n}\right)$, is the probability of the event that $x_{1}, \ldots, x_{n}$ are connected through $\mathscr{H}_{\lambda}$ (that is, $x_{i} \leftrightarrow x_{j}$ through $\mathscr{H}_{\lambda}$ for any $\left.i, j \in\{1,2, \ldots, n\}\right)$. 
For $x \in \mathbb{R}^{d}$, if there is a Poisson point of $\mathscr{H}_{\lambda}$ at $x$, we say that $x$ is occupied (otherwise, we say that $x$ is vacant). We denote the cluster containing the Poisson point $x$ by $C(x)$.

The statements of our main results for continuum percolation are as follows.

Theorem 1.1. Let $X$ be a nonnegative measurable function of $\mathscr{H}_{\lambda} \cap \Lambda$ for some bounded Borel set $\Lambda \subseteq \mathbb{R}^{d}$. Suppose that, for any nonhomogeneous Poisson process with intensity function $g(x)$ that satisfies $0<g(x) \leq \lambda_{0}$ (for some fixed $\lambda_{0} \in \mathbb{R}$ and any $x \in \Lambda$ ), any $m \geq 1$, and any nonempty $\Delta \subseteq \Lambda$, we have

$$
\limsup _{m \rightarrow \infty} \sqrt[m]{\frac{\mathrm{E}_{g}\left(X \mid B_{m}(\Delta)\right)}{m !}}=0,
$$

where $B_{m}(\Delta)$ denotes the event that $\Delta$ contains exactly $m$ Poisson points, $m=0,1, \ldots$ Then, for any homogeneous Poisson process $\mathscr{H}_{\lambda}$ with intensity $\lambda \in\left(0, \lambda_{0}\right)$, we have

$$
\frac{\mathrm{dE}_{\lambda} X}{\mathrm{~d} \lambda}=\frac{1}{\lambda} \mathrm{E}_{\lambda} Y, \quad \text { with } Y(w)=\sum_{x \in w \cap \Lambda}\left[X(w)-X\left(w_{x}\right)\right] \quad \text { for } w \in \Omega,
$$

where $w_{x}$ denotes $w \backslash\{x\}$, i.e. $w_{x}$ is $w$ with $x$ deleted.

Remark 1.1. If (1.1) is replaced by

$$
\limsup _{m \rightarrow \infty} \sqrt[m]{\frac{\mathrm{E}_{g}\left(X \mid B_{m}(\Delta)\right)}{m !}} \leq M \quad \text { for some fixed } M \in(0, \infty),
$$

then Theorem 1.1 holds with $\lambda_{0}$ replaced by $1 / M \ell(\Lambda)$ by a similar argument.

Remark 1.2. The conditions which lead to Theorem 1.1 may seem a little complicated, but for most random variables which play important roles in continuum percolation, these conditions are satisfied. For example, they are satisfied for the number of Poisson points in $\Lambda$ and the number of clusters in $\Lambda$. (See Section 3 for a strict definition of the number of clusters.)

Let $C\left(\mathbf{0}, \mathscr{H}_{\lambda, 0}\right)$ be the cluster of $\mathbf{0}$ in the graph $G\left(\mathscr{H}_{\lambda, 0}, 1\right)$ (note that a Poisson point is inserted at the origin). A key quantity in our analysis is

$$
f \equiv f(\lambda):=\mathrm{E}_{\lambda}\left(\left|C\left(\mathbf{0}, \mathscr{H}_{\lambda, 0}\right)\right|^{-1}\right)=\sum_{k=1}^{\infty} \frac{1}{k} p_{k}(\lambda) .
$$

Note that one can also define $f$ in terms of the Palm measure (for more details, see pages 39 and 117 of [14]). The function $f$ represents the mean number of clusters per Poisson point (or free energy in statistical mechanics).

Theorem 1.2. In the continuum percolation model $G\left(\mathscr{H}_{\lambda}, 1\right)$, the following two statements are equivalent.

(a) For $\lambda$ in $(0, \infty)$, either there is no infinite cluster with probability 1 or there is exactly one infinite cluster with probability 1.

(b) $f$ is a continuously differentiable function of $\lambda$ on $(0, \infty)$.

Remark 1.3. Since the continuous differentiability of $f$ has been proved in Theorem (3.6) of [2], Theorem 1.2 gives a new proof for uniqueness of the infinite cluster. (See, e.g. [8] for a traditional proof.) 
Theorem 1.3. If the infinite cluster is unique then, for any fixed $n$ and fixed $x_{1}, \ldots, x_{n}$, $\tau\left(x_{1}, \ldots, x_{n}\right)$ depends continuously on $\lambda$.

We denote by $N^{0}(x)$ the number of distinct clusters to which the Poisson neighbors of $x$ (i.e. Poisson points in the unit-radius ball with center $x$ ) belong, after $x$ is set to be vacant. We define $N^{+}(x)$ similarly except that at most one infinite cluster is counted, i.e.

$$
N^{+}(x)=N^{0}(x)-\left[N^{\#}(x)-1\right] I\left(N^{\#}(x) \geq 1\right),
$$

where $N^{\#}(x)$ is the number of distinct infinite clusters to which the Poisson neighbors of $x$ belong, after $x$ is set to be vacant, and $I(\cdot)$ denotes the indicator function. Let $\theta$ be the volume (Lebesgue measure) of the unit ball.

Theorem 1.4. Let $f$ be defined by (1.2). Then $\lambda f+\theta \lambda^{2} / 2$ is a convex function of $\lambda$, and so $\lambda f+\theta \lambda^{2} / 2$ has one-sided derivatives for all $\lambda$ in $(0, \infty)$. They are given by

$$
\begin{aligned}
& \frac{\mathrm{d}\left(\lambda f+\theta \lambda^{2} / 2\right)}{\mathrm{d} \lambda}(\lambda-0)=1+\lambda \theta-\mathrm{E}_{\lambda} N^{0}(\mathbf{0}), \\
& \frac{\mathrm{d}\left(\lambda f+\theta \lambda^{2} / 2\right)}{\mathrm{d} \lambda}(\lambda+0)=1+\lambda \theta-\mathrm{E}_{\lambda} N^{+}(\mathbf{0}) .
\end{aligned}
$$

Thus, the derivative of $\lambda f+\theta \lambda^{2} / 2$ exists at some $\lambda$ in $(0, \infty)$ if and only if, for that value of $\lambda$,

$$
\mathrm{E}_{\lambda}\left(\left[N^{\#}(\mathbf{0})-1\right] I\left(N^{\#}(\mathbf{0}) \geq 1\right)\right)=0 .
$$

Remark 1.4. The basic continuum percolation model readily lends itself to generalizations, such as balls of random radius. Our results about Russo's formula and the continuity of connectivity functions still hold for a random radius by the same arguments.

\section{The proof of Russo's formula}

In this section we prove Theorem 1.1 and present a corollary of it. An important step in the proof of Theorem 1.1 is the following lemma which will be used several times in this paper.

Lemma 2.1. Let $X$ be a measurable function of $\mathscr{H}_{\lambda} \cap \Lambda$ for some bounded Borel set $\Lambda \subseteq \mathbb{R}^{d}$, and, for some fixed $\lambda_{0}>0$,

$$
\limsup _{m \rightarrow \infty} \sqrt[m]{\frac{\mathrm{E}_{\lambda_{0}}\left(|X| \mid B_{m}(\Lambda)\right)}{m !}}=0,
$$

where $B_{m}(\Lambda)$ denotes the event that $\Lambda$ contains exactly $m$ Poisson points, $m=0,1, \ldots$ Then $\mathrm{E}_{\lambda} X$ is an infinitely differentiable function of $\lambda$ on $(0, \infty)$.

Proof. By conditioning on $B_{m}(\Lambda)$ we obtain

$$
\begin{aligned}
\mathrm{E}_{\lambda} X & =\sum_{m=0}^{\infty} \mathrm{E}_{\lambda}\left(X \mid B_{m}(\Lambda)\right) \mathrm{P}_{\lambda}\left(B_{m}(\Lambda)\right) \\
& =\sum_{m=0}^{\infty} \mathrm{E}_{\lambda}\left(X \mid B_{m}(\Lambda)\right) \frac{1}{m !}(\lambda \ell(\Lambda))^{m} \mathrm{e}^{-\lambda \ell(\Lambda)} \\
& =\mathrm{e}^{-\lambda \ell(\Lambda)} \sum_{m=0}^{\infty} \mathrm{E}_{\lambda}\left(X \mid B_{m}(\Lambda)\right) \frac{1}{m !}(\lambda \ell(\Lambda))^{m} .
\end{aligned}
$$


Note that the conditional expectations $\mathrm{E}_{\lambda}\left(X \mid B_{m}(\Lambda)\right)$ do not depend on $\lambda$ since the number of Poisson points in the set $\Lambda$ is fixed. Moreover,

$$
\begin{aligned}
\limsup _{m \rightarrow \infty} \sqrt[m]{\frac{\left|\mathrm{E}_{\lambda}\left(X \mid B_{m}(\Lambda)\right)\right|}{m !}} & =\limsup _{m \rightarrow \infty} \sqrt[m]{\frac{\left|\mathrm{E}_{\lambda_{0}}\left(X \mid B_{m}(\Lambda)\right)\right|}{m !}} \\
& \leq \limsup _{m \rightarrow \infty} \sqrt[m]{\frac{\mathrm{E}_{\lambda_{0}}\left(|X| \mid B_{m}(\Lambda)\right)}{m !}} \\
& =0 .
\end{aligned}
$$

Viewing the sum in (2.1) as a power series in $\lambda$, by (2.2) and the root test for power series (see, e.g. Theorem 3.39 of [12]), the radius of convergence for this series is $+\infty$. By the differentiability theorem for power series (see Theorem 8.1 and its corollary in [12]), $\mathrm{E}_{\lambda} X$ is an infinitely differentiable function of $\lambda$.

Proof of Theorem 1.1. By Lemma 2.1, $\mathrm{E}_{\lambda} X$ is an infinitely differentiable function of $\lambda$ on $(0, \infty)$. We follow the basic setup in the proof of Theorem 2.2 of [15]. Since $\Lambda$ is a bounded Borel subset of $\mathbb{R}^{d}$, there exists a sequence $D_{n}$ of partitions of the set $\Lambda$ into disjoint Borel subsets $\Delta_{n, 1}, \Delta_{n, 2}, \ldots, \Delta_{n, k_{n}}$ such that the diameter of the partitions

$$
d\left(D_{n}\right):=\max _{i=1, \ldots, k_{n}} \operatorname{diam}\left(\Delta_{n, i}\right)
$$

tends to 0 as $n \rightarrow \infty$. Then, by the continuity of the Lebesgue measure we have

$$
\max _{i=1, \ldots, k_{n}} \ell\left(\Delta_{n, i}\right) \rightarrow 0 \quad \text { as } n \rightarrow \infty .
$$

Consider the following family of functions $g_{n}$ on $\Lambda$ :

$$
g_{n}(x)=\sum_{i=1}^{k_{n}} \lambda_{i} I_{\Delta_{n, i}}(x) \quad \text { for } x \in \Lambda \text {. }
$$

Here $\lambda_{1}, \lambda_{2}, \ldots, \lambda_{k_{n}}$ are positive parameters less than or equal to $\lambda_{0}$ and

$$
I_{\Delta_{n, i}}(x)= \begin{cases}1 & \text { if } x \in \Delta_{n, i} \\ 0 & \text { otherwise }\end{cases}
$$

We denote by $\mathrm{P}_{\bar{\lambda}}$, where $\bar{\lambda}=\left(\lambda_{1}, \lambda_{2}, \ldots, \lambda_{k_{n}}\right)$, the probability measure associated with the nonhomogeneous Poisson process on $\Lambda$ with intensity function $g_{n}$. Obviously, $\mathrm{P}_{\bar{\lambda}}$ coincides with $\mathrm{P}_{\lambda}$ if $\lambda_{1}=\lambda_{2}=\cdots=\lambda_{k_{n}}=\lambda$.

Fix $n$ for the moment, sufficiently large so that $\lambda_{i} \ell\left(\Delta_{i}\right)<\frac{1}{2}$ for all $i$ (which we may do by (2.3)), where, to ease notation, we have omitted the index $n$, which we do for the rest of this proof. Denote by $B_{m}\left(\Delta_{i}\right)$ the event that $\Delta_{i}$ contains exactly $m$ Poisson points, $m=0,1, \ldots$ Similarly to the proof of Lemma 2.1, we have

$$
\begin{aligned}
\frac{\partial \mathrm{E}_{\bar{\lambda}} X}{\partial \lambda_{i}} & =\frac{\partial}{\partial \lambda_{i}} \sum_{m=0}^{\infty} \mathrm{E}_{\bar{\lambda}}\left(X \mid B_{m}\left(\Delta_{i}\right)\right) \mathrm{P}_{\bar{\lambda}}\left(B_{m}\left(\Delta_{i}\right)\right) \\
& =\frac{\partial}{\partial \lambda_{i}} \sum_{m=0}^{\infty} \mathrm{E}_{\bar{\lambda}}\left(X \mid B_{m}\left(\Delta_{i}\right)\right) \frac{1}{m !}\left(\lambda_{i} \ell\left(\Delta_{i}\right)\right)^{m} \mathrm{e}^{-\lambda_{i} \ell\left(\Delta_{i}\right)} \\
& =\frac{\partial}{\partial \lambda_{i}}\left[\mathrm{e}^{-\lambda_{i} \ell\left(\Delta_{i}\right)} \sum_{m=0}^{\infty} \mathrm{E}_{\bar{\lambda}}\left(X \mid B_{m}\left(\Delta_{i}\right)\right) \frac{1}{m !}\left(\lambda_{i} \ell\left(\Delta_{i}\right)\right)^{m}\right]
\end{aligned}
$$


Note that the conditional expectations $\mathrm{E}_{\bar{\lambda}}\left(X \mid B_{m}\left(\Delta_{i}\right)\right)$ do not depend on $\lambda_{i}$ and

$$
\limsup _{m \rightarrow \infty} \sqrt[m]{\frac{\left|\mathrm{E}_{\bar{\lambda}}\left(X \mid B_{m}\left(\Delta_{i}\right)\right)\right|}{m !}}=\limsup _{m \rightarrow \infty} \sqrt[m]{\frac{\mathrm{E}_{\bar{\lambda}}\left(X \mid B_{m}\left(\Delta_{i}\right)\right)}{m !}}=0
$$

by the conditions in the theorem. Thus, if we view the sum in (2.4) as a power series in $\lambda_{i}$ then, for $\lambda_{i} \in(0, \infty)$, by the differentiability theorem for power series (see Theorem 8.1 and its corollary in [12]), (2.4) equals

$$
\begin{aligned}
& -\ell\left(\Delta_{i}\right) \mathrm{E}_{\bar{\lambda}}\left(X \mid B_{0}\left(\Delta_{i}\right)\right) \mathrm{e}^{-\lambda_{i} \ell\left(\Delta_{i}\right)} \\
& +\sum_{m=1}^{\infty}\left[\mathrm{E}_{\bar{\lambda}}\left(X \mid B_{m}\left(\Delta_{i}\right)\right)\left(m \lambda_{i}^{m-1}\left(\ell\left(\Delta_{i}\right)\right)^{m}-\lambda_{i}^{m}\left(\ell\left(\Delta_{i}\right)\right)^{m+1}\right) \frac{1}{m !} \mathrm{e}^{-\lambda_{i} \ell\left(\Delta_{i}\right)}\right] .
\end{aligned}
$$

Furthermore, (1.1) implies that there exists an $M \in \mathbb{R}^{+}$such that

$$
\frac{\mathrm{E}_{\bar{\lambda}}\left(X \mid B_{m}\left(\Delta_{i}\right)\right)}{m !} \leq M
$$

for any $m \geq 1$. Recalling that we pick $n$ large enough such that $\lambda_{i} \ell\left(\Delta_{i}\right)<\frac{1}{2}$ for all $i$, we have (note that $X \geq 0$ )

$$
\begin{aligned}
& \left|\sum_{m=2}^{\infty} \mathrm{E}_{\bar{\lambda}}\left(X \mid B_{m}\left(\Delta_{i}\right)\right) m \lambda_{i}^{m-1}\left(\ell\left(\Delta_{i}\right)\right)^{m} \frac{1}{m !} \mathrm{e}^{-\lambda_{i} \ell\left(\Delta_{i}\right)}\right| \\
& \quad=\mathrm{e}^{-\lambda_{i} \ell\left(\Delta_{i}\right)} \lambda_{i}\left(\ell\left(\Delta_{i}\right)\right)^{2} \sum_{m=0}^{\infty} \mathrm{E}_{\bar{\lambda}}\left(X \mid B_{m+2}\left(\Delta_{i}\right)\right)\left(\lambda_{i} \ell\left(\Delta_{i}\right)\right)^{m} \frac{1}{(m+1) !} \\
& \quad \leq \mathrm{e}^{-\lambda_{i} \ell\left(\Delta_{i}\right)} \lambda_{i}\left(\ell\left(\Delta_{i}\right)\right)^{2} \sum_{m=0}^{\infty} M(m+2)\left(\lambda_{i} \ell\left(\Delta_{i}\right)\right)^{m} \\
& \quad=\mathrm{e}^{-\lambda_{i} \ell\left(\Delta_{i}\right)} \lambda_{i}\left(\ell\left(\Delta_{i}\right)\right)^{2} M \frac{2-\lambda_{i} \ell\left(\Delta_{i}\right)}{\left(1-\lambda_{i} \ell\left(\Delta_{i}\right)\right)^{2}} \\
& \leq 8 M \lambda_{0}\left(\ell\left(\Delta_{i}\right)\right)^{2}
\end{aligned}
$$

where we have used the fact that $\lambda_{i} \leq \lambda_{0}$ for all $i$ in the last inequality.

Similarly, we can prove that

$$
\left|\sum_{m=1}^{\infty} \mathrm{E}_{\bar{\lambda}}\left(X \mid B_{m}\left(\Delta_{i}\right)\right)\left(\lambda_{i}^{m}\left(\ell\left(\Delta_{i}\right)\right)^{m+1}\right) \frac{1}{m !} \mathrm{e}^{-\lambda_{i} \ell\left(\Delta_{i}\right)}\right| \leq 2 M \lambda_{0}\left(\ell\left(\Delta_{i}\right)\right)^{2} .
$$

Therefore, (2.4), (2.5), (2.6), and (2.7) give

$$
\frac{\partial \mathrm{E}_{\bar{\lambda}} X}{\partial \lambda_{i}}=\frac{1}{\lambda_{i}} \mathrm{E}_{\bar{\lambda}}\left(X I\left(B_{1}\left(\Delta_{i}\right)\right)\right)-\mathrm{E}_{\bar{\lambda}}\left(X I\left(B_{0}\left(\Delta_{i}\right)\right)\right) \ell\left(\Delta_{i}\right)+C_{i}\left(\ell\left(\Delta_{i}\right)\right)^{2},
$$

where $\left|C_{i}\right| \leq 10 M \lambda_{0}$.

Next, we define a random variable $X_{[i]}$ as $X_{[i]}(w)=X\left(w_{[i]}\right)$, where $w_{[i]}=w \backslash \Delta_{i}$. Since $X_{[i]}$ is independent of whether or not Poisson points exist in $\Delta_{i}$, we have

$$
\mathrm{E}_{\bar{\lambda}}\left(X_{[i]} \mid B_{1}\left(\Delta_{i}\right)\right)=\mathrm{E}_{\bar{\lambda}}\left(X_{[i]} \mid B_{0}\left(\Delta_{i}\right)\right)=\mathrm{E}_{\bar{\lambda}} X_{[i]} .
$$


Thus,

$$
\mathrm{E}_{\bar{\lambda}}\left(X_{[i]} \mid B_{1}\left(\Delta_{i}\right)\right) \mathrm{P}_{\bar{\lambda}}\left(B_{1}\left(\Delta_{i}\right)\right)=\lambda_{i} \ell\left(\Delta_{i}\right) \mathrm{E}_{\bar{\lambda}}\left(X_{[i]} \mid B_{0}\left(\Delta_{i}\right)\right) \mathrm{P}_{\bar{\lambda}}\left(B_{0}\left(\Delta_{i}\right)\right),
$$

and, thus,

$$
\frac{1}{\lambda_{i}} \mathrm{E}_{\bar{\lambda}}\left(X_{[i]} I\left(B_{1}\left(\Delta_{i}\right)\right)\right)=\mathrm{E}_{\bar{\lambda}}\left(X_{[i]} I\left(B_{0}\left(\Delta_{i}\right)\right)\right) \ell\left(\Delta_{i}\right) .
$$

Noting that $X=X-X_{[i]}+X_{[i]}$ and $\mathrm{E}_{\bar{\lambda}}\left(\left(X-X_{[i]}\right) I\left(B_{0}\left(\Delta_{i}\right)\right)\right)=0$, and substituting (2.9) into (2.8), we deduce that

$$
\frac{\partial \mathrm{E}_{\bar{\lambda}} X}{\partial \lambda_{i}}=\frac{1}{\lambda_{i}} \mathrm{E}_{\bar{\lambda}}\left(\left(X-X_{[i]}\right) I\left(B_{1}\left(\Delta_{i}\right)\right)\right)+C_{i}\left(\ell\left(\Delta_{i}\right)\right)^{2} .
$$

Set

$$
Y_{i}(w)=\sum_{x \in w \cap \Delta_{i}}\left[X(w)-X\left(w_{x}\right)\right],
$$

with the convention that an empty sum is 0 . Recalling the definition of $Y$ in Theorem 1.1, we obtain

$$
\mathrm{E}_{\lambda} Y=\sum_{i=1}^{k_{n}} \mathrm{E}_{\lambda} Y_{i}
$$

If we pick $n$ large enough such that $\lambda \ell\left(\Delta_{i}\right)<\frac{1}{2}$ for all $i$ (which we may do since $\lambda<\lambda_{0}$ ), then, for some $\left|\bar{C}_{i}\right| \leq 8 M \lambda_{0}^{2}$, we have

$$
\begin{aligned}
\mathrm{E}_{\lambda} Y_{i}= & \sum_{m=0}^{\infty} \mathrm{E}_{\lambda}\left(Y_{i} \mid B_{m}\left(\Delta_{i}\right)\right) \mathrm{P}_{\lambda}\left(B_{m}\left(\Delta_{i}\right)\right) \\
= & \mathrm{E}_{\lambda}\left(Y_{i} I\left(B_{0}\left(\Delta_{i}\right)\right)\right)+\mathrm{E}_{\lambda}\left(Y_{i} I\left(B_{1}\left(\Delta_{i}\right)\right)\right) \\
& +\sum_{m=2}^{\infty} \mathrm{E}_{\lambda}\left(Y_{i} \mid B_{m}\left(\Delta_{i}\right)\right) \mathrm{P}_{\lambda}\left(B_{m}\left(\Delta_{i}\right)\right) \\
= & \mathrm{E}_{\lambda}\left(Y_{i} I\left(B_{1}\left(\Delta_{i}\right)\right)\right)+\bar{C}_{i}\left(\ell\left(\Delta_{i}\right)\right)^{2} \\
= & \mathrm{E}_{\lambda}\left(\left(X-X_{[i]}\right) I\left(B_{1}\left(\Delta_{i}\right)\right)\right)+\bar{C}_{i}\left(\ell\left(\Delta_{i}\right)\right)^{2},
\end{aligned}
$$

where (2.12) follows because $\mathrm{E}_{\lambda}\left(Y_{i} I\left(B_{0}\left(\Delta_{i}\right)\right)\right)=0$ by the definition of $Y_{i}$ and the same reasoning as in (2.6) (note that $\left|\mathrm{E}_{\lambda}\left(Y_{i} \mid B_{m}\left(\Delta_{i}\right)\right)\right| \leq m \mathrm{E}_{\lambda}\left(X \mid B_{m}\left(\Delta_{i}\right)\right)$ by the nonnegativity of $X$ ).

Substituting (2.13) into (2.11) and taking limits on both sides of (2.11) yields

$$
\mathrm{E}_{\lambda} Y=\lim _{n \rightarrow \infty} \sum_{i=1}^{k_{n}}\left[\mathrm{E}_{\lambda}\left(\left(X-X_{[i]}\right) I\left(B_{1}\left(\Delta_{i}\right)\right)\right)+\bar{C}_{i}\left(\ell\left(\Delta_{i}\right)\right)^{2}\right] .
$$

Moreover, by (2.3) we have

$$
\sum_{i=1}^{k_{n}}\left(\ell\left(\Delta_{i}\right)\right)^{2} \leq \max _{i=1, \ldots, k_{n}} \ell\left(\Delta_{i}\right) \sum_{i=1}^{k_{n}} \ell\left(\Delta_{i}\right)=\ell(\Lambda) \max _{i=1, \ldots, k_{n}} \ell\left(\Delta_{i}\right) \rightarrow 0 \quad \text { as } n \rightarrow \infty
$$


Thus,

$$
\lim _{n \rightarrow \infty} \sum_{i=1}^{k_{n}} C_{i}\left(\ell\left(\Delta_{i}\right)\right)^{2}=0=\lim _{n \rightarrow \infty} \sum_{i=1}^{k_{n}} \bar{C}_{i}\left(\ell\left(\Delta_{i}\right)\right)^{2},
$$

since $\left|C_{i}\right| \leq 10 M \lambda_{0}$ and $\left|\bar{C}_{i}\right| \leq 8 M \lambda_{0}^{2}$.

Finally, if $\lambda_{1}=\lambda_{2}=\cdots=\lambda_{k_{n}}=\lambda$ then $\mathrm{P}_{\lambda}$ and $\mathrm{E}_{\lambda}$ respectively coincide with $\mathrm{P}_{\bar{\lambda}}$ and $\mathrm{E}_{\bar{\lambda}}$. By the chain rule,

$$
\begin{aligned}
\frac{\mathrm{dE}_{\lambda} X}{\mathrm{~d} \lambda} & =\left.\frac{\mathrm{dE}_{\bar{\lambda}} X}{\mathrm{~d} \lambda}\right|_{\bar{\lambda}=(\lambda, \lambda, \ldots, \lambda)} \\
& =\left.\sum_{i=1}^{k_{n}} \frac{\partial \mathrm{E}_{\bar{\lambda}} X}{\partial \lambda_{i}}\right|_{\bar{\lambda}=(\lambda, \lambda, \ldots, \lambda)} \\
& =\sum_{i=1}^{k_{n}}\left[\left.\frac{1}{\lambda_{i}} \mathrm{E}_{\bar{\lambda}}\left(\left(X-X_{[i]}\right) I\left(B_{1}\left(\Delta_{i}\right)\right)\right)\right|_{\bar{\lambda}=(\lambda, \ldots, \lambda)}+C_{i}\left(\ell\left(\Delta_{i}\right)\right)^{2}\right] \\
& =\frac{1}{\lambda} \mathrm{E}_{\lambda} Y,
\end{aligned}
$$

where (2.16) follows from (2.10), and (2.17) follows from taking limits on both sides of (2.16), (2.14), and (2.15).

In the rest of this section, we will give a corollary of Theorem 1.1. This corollary gives the formula for the derivative of the probability of a certain kind of event. In fact, one can also get this formula as a special case of Theorem 2.1 of [15]; see also Lemma 1 of [4].

For two configurations $w^{1}, w^{2} \in \Omega$, we define a partial ordering ' $\preceq$ ' by $w^{1} \preceq w^{2}$ if and only if $w^{1} \subseteq w^{2}$. An event $A \in \mathcal{F}$ is said to be increasing if, for every $w^{1} \preceq w^{2}$, $I(A)\left(w^{1}\right) \leq I(A)\left(w^{2}\right)$. (Recall that $I(A)$ is the indicator function of the event $A$.)

A point $x \in \mathbb{R}$ is called (+)pivotal for an event $A$ in configuration $w$ if $x \in w$ and $w \in A$, but $w_{x} \notin A$. Denote by $N(A)$ the random variable such that $N(A)(w)$ equals the number of (+)pivotal Poisson points for $(A, w)$.

Corollary 2.1. Suppose that $A$ is an increasing event and that $I(A)$ is a measurable function of $\mathscr{H}_{\lambda} \cap \Lambda$ for some bounded Borel set $\Lambda \subseteq \mathbb{R}^{d}$. Then

$$
\frac{\mathrm{d}}{\mathrm{d} \lambda} \mathrm{P}_{\lambda}(A)=\mathrm{E}_{\lambda} N(A)
$$

Proof. Let $X=I(A)$ in Theorem 1.1. Then $X$ satisfies all the conditions of Theorem 1.1. So we obtain

$$
\frac{\mathrm{d}}{\mathrm{d} \lambda} \mathrm{E}_{\lambda}(I(A))=\mathrm{E}_{\lambda} Y, \quad Y(w)=\sum_{x \in w \cap \Lambda}\left[I(A)(w)-I(A)\left(w_{x}\right)\right] .
$$

Therefore, (2.18) follows since $Y=N(A)$.

\section{The connectivity functions and the mean number of clusters per Poisson point}

In this section we will prove Theorems 1.3 and 1.4.

For any $B_{k}:=[-k / 2, k / 2]^{d}$, where $k \in \mathbb{N} \cup\{0\}$, we define clusters according to two different boundary conditions: free and wired. The free or 0 -cluster of a Poisson point $x$, 
denoted by $C^{0}\left(x, B_{k}\right)$, is the cluster of $x$ obtained when all points outside of $B_{k}$ are set to be vacant (i.e. there is no Poisson point outside of $B_{k}$ ). The wired or +-cluster of a Poisson point $x$, denoted by $C^{+}\left(x, B_{k}\right)$, is the cluster obtained when all points outside $B_{k}$ are set to be occupied (i.e. $y$ is a Poisson point for any $y \notin B_{k}$ ). Note that if $x$ is not a Poisson point then both $C^{0}\left(x, B_{k}\right)$ and $C^{+}\left(x, B_{k}\right)$ are empty.

The above definitions have the following properties.

(P1) For $x$ in $B_{k}, C^{0}\left(x, B_{k}\right)$ and $C^{+}\left(x, B_{k}\right)$ depend only on the number of Poisson points and their positions in $B_{k}$.

(P2) For $x$ in $B_{k}, C^{0}\left(x, B_{k}\right)$ is always finite, while $C^{+}\left(x, B_{k}\right)$ is infinite exactly when both $x$ is a Poisson point and $x \leftrightarrow B_{k}^{\mathrm{c}}$ (the complement of $B_{k}$ ).

(P3) Two Poisson points in $B_{k}$ are in the same 0-cluster if they are connected within $B_{k}$, whereas they are in the same +-cluster if they are either connected in $B_{k}$ or both are connected to $B_{k}^{c}$.

(P4) For a given point $x \in B_{k}, C^{0}\left(x, B_{k}\right)$ is an increasing function of $k$, while $C^{+}\left(x, B_{k}\right)$ is decreasing in $k$.

In the following we will use an asterisk to denote either 0 or + . Let $M^{0}\left(B_{k}\right)$ and $M^{+}\left(B_{k}\right)$ denote the number of finite clusters after all points in $B_{k}^{\mathrm{c}}$ are set to be vacant or, respectively, occupied. Then, we have

$$
M^{*}\left(B_{k}\right)(w)=\sum_{x \in w \cap B_{k}}\left|C^{*}\left(x, B_{k}\right)\right|^{-1} \quad \text { for } w \in \Omega .
$$

Define

$$
f_{B_{k}}^{*}:=\lambda^{-1} \ell\left(B_{k}\right)^{-1} \mathrm{E}_{\lambda} M^{*}\left(B_{k}\right) .
$$

We have the following lemma.

Lemma 3.1. For either choice of boundary conditions, we have

$$
\lim _{k \rightarrow \infty} f_{B_{2^{k}}}^{*}=f=\mathrm{E}_{\lambda}\left(\left|C\left(\mathbf{0}, \mathscr{H}_{\lambda, 0}\right)\right|^{-1}\right) .
$$

Remark 3.1. Note that we restrict the limit to a subsequence of $\mathbb{N}$ (i.e. $\left\{2^{k}\right\}, k \in \mathbb{N}$ ) in order to obtain the monotone property (see (3.1) below). We will use this method again in (3.9) below.

Proof of Lemma 3.1. By Palm theory (see, e.g. Theorem 1.6 of [10]),

$$
f_{B_{k}}^{*}=\lambda^{-1} \ell\left(B_{k}\right)^{-1} \mathrm{E}_{\lambda} M^{*}\left(B_{k}\right)=\mathrm{E}_{\lambda}\left|C^{*}\left(U_{k}, U_{k} \cup \mathscr{H}_{\lambda}, B_{k}\right)\right|^{-1},
$$

where $U_{k}$ is a uniformly distributed random variable on $B_{k}$ independent of $\mathscr{H}_{\lambda}$, and

$$
\left|C^{*}\left(U_{k}, U_{k} \cup \mathscr{H}_{\lambda}, B_{k}\right)\right|^{-1}=\frac{1}{\ell\left(B_{k}\right)} \int_{B_{k}}\left|C^{*}\left(x,\{x\} \cup \mathscr{H}_{\lambda}, B_{k}\right)\right|^{-1} \mathrm{~d} x,
$$

where $C^{*}\left(x,\{x\} \cup \mathscr{H}_{\lambda}, B_{k}\right)$ is $C^{*}\left(x, B_{k}\right)$ after a Poisson point is inserted at $x$, that is, $C^{*}(x,\{x\} \cup$ $\left.\mathscr{H}_{\lambda}, B_{k}\right)(w)=C^{*}\left(x, B_{k}\right)(w \cup\{x\})$ for $w \in \Omega$. 
We partition $B_{2^{k+1}}$ into $2^{d}$ boxes $B_{2^{k}}^{1}, B_{2^{k}}^{2}, \ldots, B_{2^{k}}^{2^{d}}$ such that each $B_{2^{k}}^{i}$ is a translation of $B_{2^{k}}$ (we do not need to consider the boundary since the Lebesgue measure of the boundary is $0)$. Then we have

$$
\begin{aligned}
\mathrm{E}_{\lambda}\left|C^{0}\left(U_{2^{k+1}}, U_{2^{k+1}} \cup \mathscr{H}_{\lambda}, B_{2^{k+1}}\right)\right|^{-1} \\
=\frac{1}{\ell\left(B_{2^{k+1}}\right)} \mathrm{E}_{\lambda} \int_{B_{2^{k+1}}}\left|C^{0}\left(x,\{x\} \cup \mathscr{H}_{\lambda}, B_{2^{k+1}}\right)\right|^{-1} \mathrm{~d} x \\
=\frac{1}{\ell\left(B_{2^{k+1}}\right)} \sum_{i=1}^{2^{d}} \mathrm{E}_{\lambda} \int_{B_{2^{k}}^{i}}\left|C^{0}\left(x,\{x\} \cup \mathscr{H}_{\lambda}, B_{2^{k+1}}\right)\right|^{-1} \mathrm{~d} x \\
\leq \frac{1}{\ell\left(B_{2^{k+1}}\right)} \sum_{i=1}^{2^{d}} \mathrm{E}_{\lambda} \int_{B_{2^{k}}^{i}}\left|C^{0}\left(x,\{x\} \cup \mathscr{H}_{\lambda}, B_{2^{k}}^{i}\right)\right|^{-1} \mathrm{~d} x \\
=\frac{\ell\left(B_{2^{k}}\right)}{\ell\left(B_{2^{k+1}}\right)} \sum_{i=1}^{2^{d}} \mathrm{E}_{\lambda}\left|C^{0}\left(U_{2^{k}}, U_{2^{k}} \cup \mathscr{H}_{\lambda}, B_{2^{k}}\right)\right|^{-1} \\
=\mathrm{E}_{\lambda}\left|C^{0}\left(U_{2^{k}}, U_{2^{k}} \cup \mathscr{H}_{\lambda}, B_{2^{k}}\right)\right|^{-1}
\end{aligned}
$$

for any $k \in \mathbb{N}$ (the inequality follows by (P4) for the $*$-cluster). Therefore, $\mathrm{E}_{\lambda} \mid C^{0}\left(U_{2^{k}}, U_{2^{k}} \cup\right.$ $\left.\mathscr{H}_{\lambda}, B_{2^{k}}\right)\left.\right|^{-1}$ decreases to $\mathrm{E}_{\lambda}\left|C^{0}\left(U_{\infty}, U_{\infty} \cup \mathscr{H}_{\lambda}, \mathbb{R}^{d}\right)\right|^{-1} \equiv f$ as $k \rightarrow \infty$.

Finally, $\mathrm{E}_{\lambda}\left|C^{+}\left(U_{2^{k}}, U_{2^{k}} \cup \mathscr{H}_{\lambda}, B_{2^{k}}\right)\right|^{-1} \uparrow \mathrm{E}_{\lambda}\left|C^{+}\left(U_{\infty}, U_{\infty} \cup \mathscr{H}_{\lambda}, \mathbb{R}^{d}\right)\right|^{-1} \equiv f$ as $k \rightarrow$ $\infty$ by a similar argument.

We will now turn to the derivatives of $f_{B_{k}}^{*}$. For $x \in B_{k}$, we define $N^{0}\left(x, B_{k}\right)$ and $N^{+}\left(x, B_{k}\right)$ to be the number of distinct (finite or infinite) clusters obtained after setting $x$ to be vacant and setting all points in $B_{k}^{\mathrm{c}}$ to be vacant or, respectively, occupied, which contain a nearest Poisson neighbor to $x$. Note that if $N^{*}\left(x, B_{k}\right)=0$ then changing $x$ from vacant to occupied increases $M^{*}\left(B_{k}\right)$ by 1 , but if $N^{*}\left(x, B_{k}\right) \geq 1$, then such a change in $x$ decreases $M^{*}\left(B_{k}\right)$ by $N^{*}\left(x, B_{k}\right)-1$.

With the definition above and according to the definition of $N^{*}(x)$ given before Theorem 1.4, we have

$$
N^{*}(x)=\lim _{k \rightarrow \infty} N^{*}\left(x, B_{k}\right) .
$$

Next, we deduce the formula for $(\mathrm{d} / \mathrm{d} \lambda)\left(\lambda f_{B_{k}}^{*}+\theta \lambda^{2} / 2\right)$ by Russo's formula in Section 1.

Lemma 3.2. For any $k \in \mathbb{N}$ and either choice of boundary conditions, $\lambda f_{B_{k}}^{*}+\theta \lambda^{2} / 2$ is a convex function of $\lambda$ on $(0, \infty)$ with

$$
\frac{\mathrm{d}}{\mathrm{d} \lambda}\left(\lambda f_{B_{k}}^{*}+\frac{\theta}{2} \lambda^{2}\right)=\lambda^{-1} \ell\left(B_{k}\right)^{-1} \mathrm{E}_{\lambda} Y+\theta \lambda,
$$

where

$$
Y(w)=\sum_{x \in w \cap B_{k}}\left[1-N^{*}\left(x, B_{k}\right)\right] \text { for } w \in \Omega .
$$

Proof. By Theorem 1.1,

$$
\begin{aligned}
\frac{\mathrm{d}}{\mathrm{d} \lambda} f_{B_{k}}^{*} & =\frac{\mathrm{d}}{\mathrm{d} \lambda}\left[\lambda^{-1} \ell\left(B_{k}\right)^{-1} \mathrm{E}_{\lambda} M^{*}\left(B_{k}\right)\right] \\
& =-\lambda^{-2} \ell\left(B_{k}\right)^{-1} \mathrm{E}_{\lambda} M^{*}\left(B_{k}\right)+\lambda^{-2} \ell\left(B_{k}\right)^{-1} \mathrm{E}_{\lambda} Y
\end{aligned}
$$


where

$$
\begin{aligned}
Y(w) & =\sum_{x \in w, x \in B_{k}}\left[M^{*}\left(B_{k}\right)(w)-M^{*}\left(B_{k}\right)\left(w_{x}\right)\right] \\
& =\sum_{x \in w, x \in B_{k}}\left[I\left(N^{*}\left(x, B_{k}\right)=0\right)+\left(1-N^{*}\left(x, B_{k}\right)\right) I\left(N^{*}\left(x, B_{k}\right) \geq 1\right)\right] \\
& =\sum_{x \in w, x \in B_{k}}\left[1-N^{*}\left(x, B_{k}\right)\right] .
\end{aligned}
$$

Thus, by (3.2) we obtain

$$
\frac{\mathrm{d}}{\mathrm{d} \lambda}\left(\lambda f_{B_{k}}^{*}+\frac{\theta}{2} \lambda^{2}\right)=f_{B_{k}}^{*}+\lambda \frac{\mathrm{d}}{\mathrm{d} \lambda} f_{B_{k}}^{*}+\theta \lambda=\lambda^{-1} \ell\left(B_{k}\right)^{-1} \mathrm{E}_{\lambda} Y+\theta \lambda .
$$

Next, we prove that $\lambda f_{B_{k}}^{*}+\theta \lambda^{2} / 2$ is a convex function of $\lambda$. For $x \in B_{k}$, let us define $N_{\mathrm{Po}}(x)$ to be the number of Poisson neighbors of $x$. Let

$$
N_{k}\left(U_{k}, U_{k} \cup \mathscr{H}_{\lambda}, B_{k}\right):=\frac{1}{\ell\left(B_{k}\right)} \int_{B_{k}} N_{k}\left(x,\{x\} \cup \mathscr{H}_{\lambda}, B_{k}\right) \mathrm{d} x,
$$

where $U_{k}$ is a uniformly distributed random variable on $B_{k}$ independent of $\mathscr{H}_{\lambda}$ and $N_{k}(x,\{x\} \cup$ $\left.\mathscr{H}_{\lambda}, B_{k}\right)=N_{\text {Po }}(x)$. Then, by Fubini's theorem (see page 407 of [3]) we have

$$
\mathrm{E}_{\lambda} N_{k}\left(U_{k}, U_{k} \cup \mathscr{H}_{\lambda}, B_{k}\right)=\lambda \theta
$$

for any $k \in \mathbb{N}$.

By (3.4), (3.3), Palm theory, (3.6), and Fubini's theorem, we obtain

$$
\begin{aligned}
\frac{\mathrm{d}}{\mathrm{d} \lambda}( & \left.\lambda f_{B_{k}}^{*}+\frac{\theta}{2} \lambda^{2}\right) \\
& =\lambda^{-1} \ell\left(B_{k}\right)^{-1} \mathrm{E}_{\lambda} Y+\theta \lambda \\
& =\mathrm{E}_{\lambda}\left(1-N^{*}\left(U_{k}, U_{k} \cup \mathscr{H}_{\lambda}, B_{k}\right)\right)+\mathrm{E}_{\lambda} N_{k}\left(U_{k}, U_{k} \cup \mathscr{H}_{\lambda}, B_{k}\right) \\
& =1+\mathrm{E}_{\lambda}\left(N_{k}\left(U_{k}, U_{k} \cup \mathscr{H}_{\lambda}, B_{k}\right)-N^{*}\left(U_{k}, U_{k} \cup \mathscr{H}_{\lambda}, B_{k}\right)\right) \\
& =1+\mathrm{E}_{\lambda}\left(\frac{1}{\ell\left(B_{k}\right)} \int_{B_{k}}\left[N_{k}\left(x,\{x\} \cup \mathscr{H}_{\lambda}, B_{k}\right)-N^{*}\left(x,\{x\} \cup \mathscr{H}_{\lambda}, B_{k}\right)\right] \mathrm{d} x\right) \\
& =1+\frac{1}{\ell\left(B_{k}\right)} \int_{B_{k}} \mathrm{E}_{\lambda}\left(N_{k}\left(x,\{x\} \cup \mathscr{H}_{\lambda}, B_{k}\right)-N^{*}\left(x,\{x\} \cup \mathscr{H}_{\lambda}, B_{k}\right)\right) \mathrm{d} x,
\end{aligned}
$$

where $N^{*}\left(U_{k}, U_{k} \cup \mathscr{H}_{\lambda}, B_{k}\right)$ is defined similarly to (3.5) and $N^{*}\left(x,\{x\} \cup \mathscr{H}_{\lambda}, B_{k}\right)$ is $N^{*}\left(x, B_{k}\right)$ after a Poisson point is inserted at $x$. Since $N_{k}\left(x,\{x\} \cup \mathscr{H}_{\lambda}, B_{k}\right)-N^{*}\left(x,\{x\} \cup \mathscr{H}_{\lambda}, B_{k}\right)$ cannot decrease when a point is changed from vacant to occupied,

$$
\mathrm{E}_{\lambda}\left(N_{k}\left(x,\{x\} \cup \mathscr{H}_{\lambda}, B_{k}\right)-N^{*}\left(x,\{x\} \cup \mathscr{H}_{\lambda}, B_{k}\right)\right)
$$

is increasing in $\lambda$ by a coupling argument (see page 28 of [9]). Therefore, (3.8) is increasing in $\lambda$, and, thus, $\lambda f_{B_{k}}^{*}+\theta \lambda^{2} / 2$ is a convex function of $\lambda$.

Theorem 1.4 is now a consequence of the previous two lemmas. 
Proof of Theorem 1.4. By Lemma 3.1 and Lemma 3.2, $\lambda f+\theta \lambda^{2} / 2$ is the limit of convex functions $\lambda f_{B_{2^{k}}}^{0}+\theta \lambda^{2} / 2$ (as $k \rightarrow \infty$ ). Furthermore, by (3.7) and (3.6),

$$
\frac{\mathrm{d}}{\mathrm{d} \lambda}\left(\lambda f_{B_{2^{k}}}^{0}+\frac{\theta}{2} \lambda^{2}\right)=1+\lambda \theta-\mathrm{E}_{\lambda}\left(N^{0}\left(U_{2^{k}}, U_{2^{k}} \cup \mathscr{H}_{\lambda}, B_{2^{k}}\right)\right) .
$$

By the same argument used for Lemma 3.1, we can prove that

$$
\mathrm{E}_{\lambda}\left(N^{0}\left(U_{2^{k}}, U_{2^{k}} \cup \mathscr{H}_{\lambda}, B_{2^{k}}\right)\right) \downarrow \mathrm{E}_{\lambda}\left(N^{0}\left(U_{\infty}, U_{\infty} \cup \mathscr{H}_{\lambda}, \mathbb{R}^{d}\right)\right) \equiv \mathrm{E}_{\lambda} N^{0}(\mathbf{0}) .
$$

By Fubini's theorem,

$$
\begin{aligned}
\mathrm{E}_{\lambda}\left(N^{0}\left(U_{2^{k}}, U_{2^{k}} \cup \mathscr{H}_{\lambda}, B_{2^{k}}\right)\right) & =\mathrm{E}_{\lambda}\left(\frac{1}{\ell\left(B_{2^{k}}\right)} \int_{B_{2^{k}}} N^{0}\left(x,\{x\} \cup \mathscr{H}_{\lambda}, B_{2^{k}}\right) \mathrm{d} x\right) \\
& =\frac{1}{\ell\left(B_{2^{k}}\right)} \int_{B_{2^{k}}} \mathrm{E}_{\lambda}\left(N^{0}\left(x,\{x\} \cup \mathscr{H}_{\lambda}, B_{2^{k}}\right)\right) \mathrm{d} x .
\end{aligned}
$$

Since $\mathrm{E}_{\lambda}\left(N^{0}\left(x,\{x\} \cup \mathscr{H}_{\lambda}, B_{2^{k}}\right)\right)$ is continuous in $\lambda$ by Lemma $2.1, \mathrm{E}_{\lambda}\left(N^{0}\left(U_{2^{k}}, U_{2^{k}} \cup \mathscr{H}_{\lambda}, B_{2^{k}}\right)\right)$ is a continuous function of $\lambda$. Moreover, by (3.6),

$$
\lambda \theta-\mathrm{E}_{\lambda} N^{0}(\mathbf{0})=\mathrm{E}_{\lambda}\left(N_{\infty}\left(\mathbf{0},\{\mathbf{0}\} \cup \mathscr{H}_{\lambda}, \mathbb{R}^{d}\right)-N^{0}\left(\mathbf{0},\{\mathbf{0}\} \cup \mathscr{H}_{\lambda}, \mathbb{R}^{d}\right)\right)
$$

is increasing in $\lambda$ (see the reason below (3.8)). Thus, $1+\lambda \theta-\mathrm{E}_{\lambda} N^{0}(\mathbf{0})$ is the increasing limit of continuous functions; therefore, it is lower semicontinuous. On the other hand, $1+\lambda \theta-$ $\mathrm{E}_{\lambda} N^{0}(\mathbf{0})$ is nondecreasing in $\lambda$, and, thus, it is left continuous.

It follows, as in the proof of Proposition 1.4 of [1], that $\lambda f+\theta \lambda^{2} / 2$ is convex with its left derivative given by (1.3). The analogous argument with 0 substituted by + and left by right yields (1.4).

From (1.3) and (1.4), we obtain the identity

$$
\frac{\mathrm{d}\left(\lambda f+\theta \lambda^{2} / 2\right)}{\mathrm{d} \lambda}(\lambda+0)-\frac{\mathrm{d}\left(\lambda f+\theta \lambda^{2} / 2\right)}{\mathrm{d} \lambda}(\lambda-0)=\mathrm{E}_{\lambda}\left(\left[N^{\#}(\mathbf{0})-1\right] I\left(N^{\#}(\mathbf{0}) \geq 1\right)\right) .
$$

This completes the proof.

Finally, we will deal with the continuity of connectivity functions. For $x_{1}, \ldots, x_{n} \in \mathbb{R}^{d}$, we define

$$
\begin{gathered}
\tau_{B_{k}}^{*}\left(x_{1}, x_{2}, \ldots, x_{n}\right):=\mathrm{P}_{\lambda}\left(x_{1}, x_{2}, \ldots, x_{n} \text { are connected through the same } * \text {-cluster of } B_{k}\right), \\
\tau^{+}\left(x_{1}, x_{2}, \ldots, x_{n}\right):=\mathrm{P}_{\lambda}\left(x_{1}, x_{2}, \ldots, x_{n}\right. \text { are connected through the same cluster or } \\
\left.C\left(x_{1}\right), \ldots, C\left(x_{n}\right) \text { are all infinite }\right),
\end{gathered}
$$

and $\tau^{0}$ denotes the ordinary connectivity function $\tau$.

Lemma 3.3. For either choice of boundary conditions and any fixed $n$,

$$
\tau_{B_{k}}^{*}\left(x_{1}, x_{2}, \ldots, x_{n}\right) \rightarrow \tau^{*}\left(x_{1}, x_{2}, \ldots, x_{n}\right) \text { as } k \rightarrow \infty .
$$

Here $\tau\left(x_{1}, x_{2}, \ldots, x_{n}\right)$ is a left continuous function of $\lambda$ while $\tau^{+}\left(x_{1}, x_{2}, \ldots, x_{n}\right)$ is right continuous. 
Proof. We follow the proof of Lemma 2.3 of [1]. For fixed $x, C^{0}\left(x, B_{k}\right)$ is increasing in $k$ while $C^{+}\left(x, B_{k}\right)$ is decreasing in $k$ and $C^{0}\left(x, B_{k}\right) \subseteq C^{+}\left(x, B_{k}\right)$. Furthermore, if $C(x)$ is finite then $C(x)=C^{*}\left(x, B_{k}\right)$ for sufficiently large $k$. If $C(x)$ is infinite then $C^{0}\left(x, B_{k}\right) \rightarrow C(x)$, while $C^{+}\left(x, B_{k}\right) \rightarrow\{y:|C(y)|=\infty\}$ as $k \rightarrow \infty$. It follows that, for fixed $x_{1}, x_{2}, \ldots, x_{n}$,

$$
\begin{array}{cc}
\tau_{B_{k}}^{0}\left(x_{1}, x_{2}, \ldots, x_{n}\right) \uparrow \tau^{0}\left(x_{1}, x_{2}, \ldots, x_{n}\right) & \text { as } k \uparrow \infty, \\
\tau_{B_{k}}^{+}\left(x_{1}, x_{2}, \ldots, x_{n}\right) \downarrow \tau^{+}\left(x_{1}, x_{2}, \ldots, x_{n}\right) & \text { as } k \uparrow \infty .
\end{array}
$$

Note that (3.10) holds for large enough $k$ such that $B_{k}$ contains $x_{1}, x_{2}, \ldots, x_{n}$.

Moreover, by Lemma $2.1, \tau_{B_{k}}^{*}\left(x_{1}, x_{2}, \ldots, x_{n}\right)$ is a continuously differentiable function of $\lambda$. Thus, $\tau^{0}\left(x_{1}, x_{2}, \ldots, x_{n}\right)$ is the increasing limit of continuous functions; therefore, $\tau^{0}\left(x_{1}, x_{2}, \ldots, x_{n}\right)$ is lower semicontinuous. On the other hand, $\tau^{0}\left(x_{1}, x_{2}, \ldots, x_{n}\right)$ is nondecreasing in $\lambda$, and, thus, $\tau^{0}\left(x_{1}, x_{2}, \ldots, x_{n}\right)$ is continuous from the left.

Similar arguments are valid for the right continuity of $\tau^{+}\left(x_{1}, x_{2}, \ldots, x_{n}\right)$.

Proof of Theorem 1.3. The uniqueness of the infinite cluster implies that

$$
\tau\left(x_{1}, \ldots, x_{n}\right)=\tau^{+}\left(x_{1}, \ldots, x_{n}\right) .
$$

Furthermore, by Lemma 3.3, $\tau\left(x_{1}, \ldots, x_{n}\right)$ is a left continuous function of $\lambda$ and $\tau^{+}\left(x_{1}, \ldots, x_{n}\right)$ is right continuous. These facts yield the required result.

\section{Proof of Theorem 1.2}

In this section we explain the few extra arguments needed to obtain Theorem 1.2 from the results of the last two sections.

Proof of Theorem 1.2. Part (a) implies part (b). If the infinite cluster is unique then, by Theorem 1.4, $\lambda f+\theta \lambda^{2} / 2$ is convex and differentiable. Thus, by Corollary 25.5.1 of [11], $\lambda f+$ $\theta \lambda^{2} / 2$ is continuously differentiable on $(0, \infty)$. Therefore, $f$ is a continuously differentiable function of $\lambda$ on $(0, \infty)$.

Part (b) implies part (a). We follow the arguments in the proof of Proposition 5.3 of [1]. Since $f$ is differentiable, $\lambda f+\theta \lambda^{2} / 2$ is differentiable. Then Theorem 1.4 implies that (1.5) is always true and, thus, that

$$
\mathrm{P}_{\lambda}\left(\left(N^{\#}(\mathbf{0})\right) \geq 2\right)=0 .
$$

To prove the implication (b) $\Rightarrow$ (a), it suffices to assume that more than one infinite cluster occurs with nonzero probability and derive a contradiction to (4.1). Under this assumption, $\mathrm{P}_{\lambda}\left(G\left(B_{k}\right)\right)$ is positive for some large $k$, where $G\left(B_{k}\right)$ is the event that at least two distinct infinite clusters occur and intersect $B_{k}$. It is easy to construct a mapping $\Phi$ on configuration $w$ in $G\left(B_{k}\right)$. The mapping $\Phi$ only changes the occupation status of points in $B_{k}$ and $\Phi\left(B_{k}\right)$ is contained in the event that $N^{\#}(\mathbf{0}) \geq 2$. However, $\mathrm{P}_{\lambda}\left(\Phi\left(B_{k}\right)\right)>0$ implies that $\mathrm{P}_{\lambda}\left(\left(N^{\#}(\mathbf{0})\right) \geq 2\right)>0$, which contradicts (4.1).

\section{Acknowledgements}

The authors would like to thank the anonymous referee for providing constructive comments and help in improving the contents of this paper. We thank Tom Kennedy for advice regarding the proof of Theorem 1.1 and help with the English presentation. This research was supported in part by the Knowledge Innovation Program of the Chinese Academy of Sciences (grant number kjcx-yw-s7) and the National Natural Science Foundation of China (grant numbers 10831006 and 10801133). 


\section{References}

[1] Aizenman, M., Kesten, H. and Newman, C. M. (1987). Uniqueness of the infinite cluster and continuity of connectivity functions for short and long range percolation. Commun. Math. Phys. 111, 505-531.

[2] Bezuidenhout, C. E., Grimmett, G. And Löffler, A. (1998). Percolation and minimal spanning trees. J. Statist. Phys. 92, 1-34.

[3] Durrett, R. (2007). Probability: Theory and Examples, 3rd edn. Duxbury Press, Belmont, CA.

[4] Franceschetti, M., Penrose, M. D. and Rosoman, T. (2010). Strict inequalities of critical probabilities on Gilbert's continuum percolation graph. Preprint. Available at http://arxiv.org/abs/1004.1596v2.

[5] Grimmett, G. (1999). Percolation, 2nd edn. Springer, Berlin.

[6] Grimmett, G. (2010). Probability on Graphs. Cambridge University Press.

[7] Margulis, G. A. (1974). Probabilistic characteristics of graphs with large connectivity. Probl. Peredachi Inf. 10, 101-108.

[8] Meester, R. AND Roy, R. (1994). Uniqueness of unbounded occupied and vacant components in Boolean models. Ann. Appl. Prob. 4, 933-951.

[9] Meester, R. ANd Roy, R. (1996). Continuum Percolation. Cambridge University Press.

[10] Penrose, M. D. (2003). Random Geometric Graphs. Oxford University Press.

[11] Rockafellar, R. T. (1970). Convex Analysis. Princeton University Press.

[12] Rudin, W. (1976). Principles of Mathematical Analysis, 3rd edn. McGraw-Hill, New York.

[13] Russo, L. (1981). On the critical percolation probabilities. Z. Wahrscheinlichkeitsth. 56, 229-237.

[14] Stoyan, D., Kendall, W. S. And Mecke, J. (1995). Stochastic Geometry and Its Applications, 2nd edn. John Wiley, Chichester.

[15] Zuev, S. A. (1993). Russo's formula for Poisson point fields and its application. Discrete Math. Appl. 3, 63-73. 